\title{
Fulminant endocarditis caused by Streptobacillus moniliformis in a young man
}

\author{
Markus Kondruweit, MD, ${ }^{\text {a }}$ Michael Weyand, MD, ${ }^{a}$ Faidi Omar Mahmoud, MD, ${ }^{a}$ Walter Geissdoerfer, PhD, ${ }^{b}$ \\ Christoph Schoerner, MD, ${ }^{\mathrm{b}}$ Dieter Ropers, MD, ${ }^{\mathrm{c}}$ Stephan Achenbach, MD, ${ }^{\mathrm{c}}$ and Thomas Strecker, MD, ${ }^{\mathrm{a}}$ Erlangen, Germany
}

$\mathrm{R}$ at bite fever caused by Streptobacillus moniliformis is a very rare infectious disease and is characterized by the abrupt onset of fever, arthralgias, polyarthritis, and skin rash. Manifestation as acute endocarditis is extremely uncommon. Here we present the successful repair of massive endocarditis with giant floating vegetations on a degenerated, insufficient aortic valve. Two months after surgery, echocardiography showed almost normal left and right ventricular function with a well-functioning aortic valve prosthesis.

\section{Clinical Summary}

A 29-year-old farmer with a history of fever, dyspnea, dizziness, and increasing somnolence was referred to our hospital. He reported having been injured on his right hand 2 weeks earlier and a feeling of progressive lethargy and weakness for the week before coming to our clinic. A transesophageal echocardiogram revealed endocarditis with giant floating vegetations on a degenerated, insufficient aortic valve and a large perivalvular abscess cavity (Figure 1, A). Left ventricular function was highly impaired, with an ejection fraction of $20 \%$. An antibiotic regimen with ampicillin, ceftriaxone, and gentamicin was initiated. Because of progressive cardiopulmonary deterioration, rapid surgical management was required.

The patient was taken to the operating theater, where cardiopulmonary bypass was performed via aorta-right atrium cannulation with moderate hypothermia. Intraoperatively, the aortic valve showed massive inflammatory vegetations on the aortic valve and a huge abscess formation near the annulus (Figure 1, B). After resection of all inflamed tissue, the aortic annulus was reconstructed, and subsequently a 23-mm St Jude Medical aortic valve prosthesis (St Jude Medical, Inc, St Paul, Minn) was implanted. Assisted by counterpulsation therapy with an intra-aortic balloon catheter, the patient was transferred to the intensive care unit in stable hemodynamic condition. Histologic examination of the resected tissue revealed recent

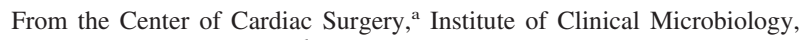
Immunology and Hygiene, ${ }^{\mathrm{b}}$ and Department of Cardiology, ${ }^{\mathrm{c}}$ FriedrichAlexander-University, Erlangen-Nuremberg, Germany.

Received for publication July 6, 2007; accepted for publication Aug 10, 2007.

Address for reprints: Thomas Strecker, MD, Center of Cardiac Surgery, Friedrich-Alexander-University Erlangen-Nuremberg, Krankenhausstrasse 12, 91054 Erlangen, Germany (E-mail: thomas.strecker@herz.imed.unierlangen.de).

J Thorac Cardiovasc Surg 2007;134:1579-80

$0022-5223 / \$ 32.00$

Copyright () 2007 by The American Association for Thoracic Surgery

doi:10.1016/j.jtcvs.2007.08.010 necrotic and inflammatory thrombotic material without evidence of malignancy. In microbiologic examination, blood cultures remained negative after 14 days of incubation. Microscopy of the explanted aortic valve revealed Gram-positive/ Gram-variable bacteria, which appeared as straight, curved, and filamentous rods (Figure 2). After 48 hours, tiny colonies grew on sheep blood agar, showing long filamentous Gram-variable rods. The bacteria were identified as Streptobacillus moniliformis by analysis of the 16S rRNA gene sequence. The identical sequence was amplified directly from the explanted valve specimen, confirming Streptobacillus moniliformis as the etiologic agent. The Streptobacillus moniliformis isolate did not grow on chocolate agar, formed typical "cotton balls" in thioglycolate broth supplemented with $10 \%$ horse serum, and was negative for catalase, oxidase, and indole. It was highly susceptible to penicillins, cephalosporins, carbapenems, aminoglycosides, quinolones, erythromycin, clindamycin, and vancomycin. Consequently, the empirical antibiotic therapy was adjusted to penicillin, fosfomycin, and gentamicin. Postoperatively, the patient had signs of septic multiorgan failure and was placed on hemofiltration for 15 days. Before the patient left the hospital 2 months after the operation, echocardiography showed almost normal right and left ventricular function, a well-functioning aortic valve prosthesis, no paravalvular leakage, and no relevant pericardial effusion.

\section{Discussion}

Rat bite fever is a very uncommon condition and is characterized by the abrupt onset of fever, rigors, arthralgias, polyarthritis, and skin rash. The bacterium Streptobacillus moniliformis has been identified as the causative agent. It is transmitted by rat bite or by ingestion of contaminated food or water. ${ }^{1}$ Manifestation as acute endocarditis is extremely uncommon, and cardiac valvular dysfunctions have been reported in $50 \%$ of cases. $^{2}$ If left untreated, rat bite fever carries a mortality rate of $10 \%$. Unfortunately, its nonspecific initial presentation combined with difficulties in culturing its causative organism produces a significant risk of delay or failure in diagnosis. ${ }^{3}$

Streptobacillus moniliformis is a fastidious facultatively anaerobic, pleomorphic, Gram-variable bacillus often seen in chains and as long unbranched filaments. It is found in the nasopharynx and oropharynx of small rodents, especially wild and laboratory rats. ${ }^{4}$ The microbiologic characterization of Streptobacillus monoiliformis by $16 \mathrm{~S}$ rDNA sequencing is an efficient tool for identification of that fastidious bacterium. ${ }^{5}$

If echocardiography confirms the clinical suspicion of endocarditis by demonstrating large vegetations on the heart valves with valve rupture or insufficiency, rapid surgical management is indicated. The currently recommended antimicrobial therapy of rat bite 

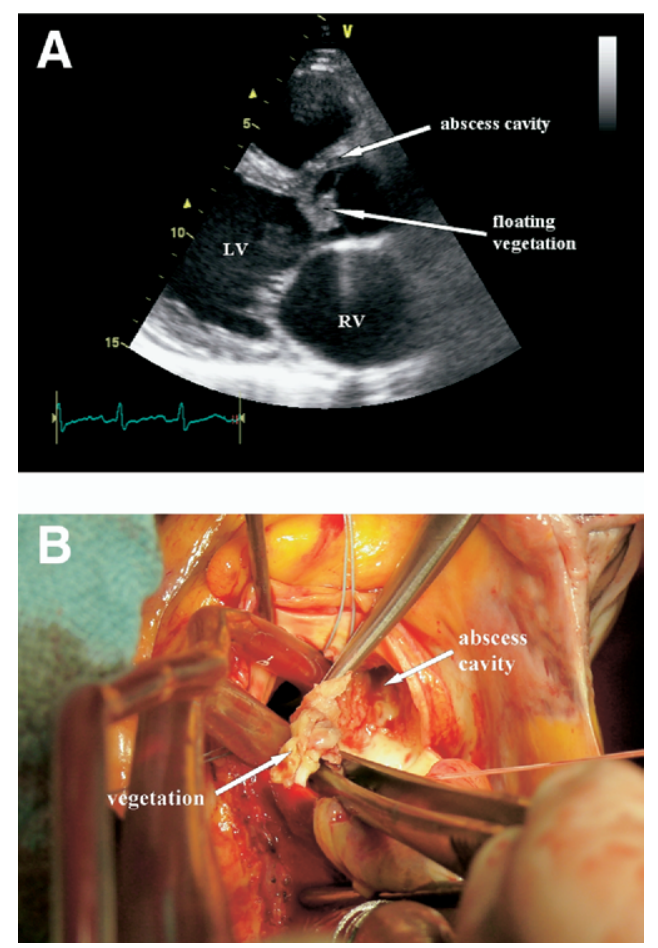

Figure 1. A, Transesophageal echocardiogram revealed a giant floating vegetation on a degenerated, insufficient aortic valve and a large perivalvular abscess cavity. The annulus was almost completely destroyed. $L V$, Left ventricle; $R V$, right ventricle. $B$, Intraoperative photograph showing the massive inflammatory vegetations on the aortic valve and the huge abscess formation near the annulus.

fever is penicillin; if the patient is allergic to penicillin, tetracycline is the second choice. ${ }^{2}$

\section{References}

1. van Nood E. Peters SH. Rat-bite fever. Neth J Med. 2005;63:319-21.
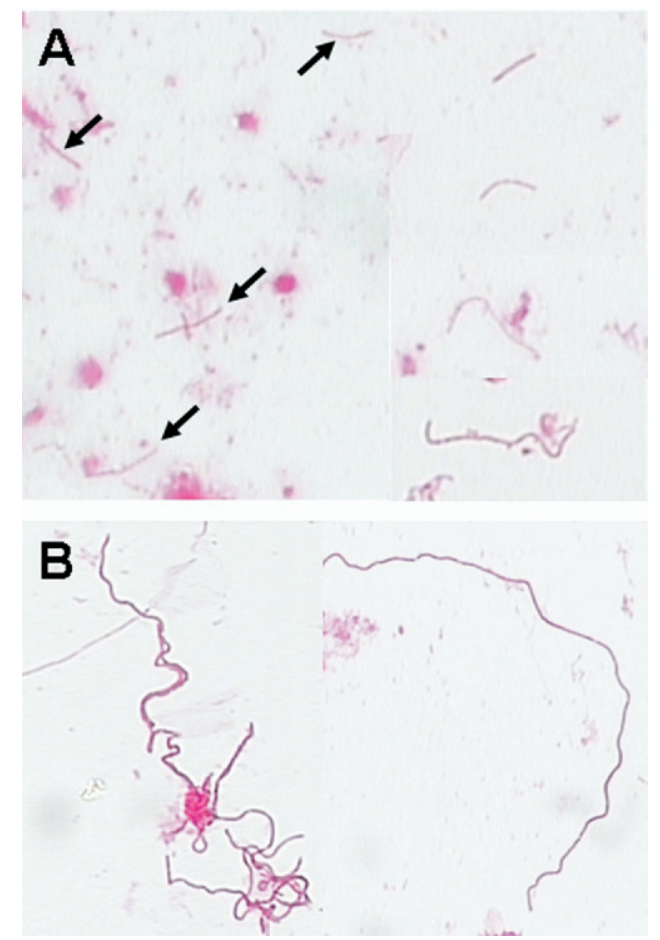

Figure 2. Microbiology: A, Gram stain of the explanted aortic valve tissue showing pleomorphic Gram-variable bacteria, which appear as straight rods (upper arrows) and curved or filamentous rods (lower arrows). B, Gram stain of primary Streptobacillus moniliformis culture after $\mathbf{4 8}$ hours of growth on sheep blood agar showing long curved filamentous bacteria.

2. Rordorf T, Züger C, Zbinden R, von Graevenitz A, Pirovino M. Streptobacillus moniliformis endocarditis in an HIV-positive patient. Infection. 2000;28:393.

3. Elliott SP. Rat bite fever and Streptobacillus moniliformis. Clin Microbiol Rev. 2007;20:13-22.

4. Balakrishnan N, Menon T, Shanmugasundaram S, Alagesan R. Streptobacillus moniliformis endocarditis. Emerg Infect Dis. 2006;12:1037-8.

5. Andre JM, Freydiere AM, Benito Y, Rousson A, Lansiaux S, Kodjo A, et al. Rat bite fever caused by Streptobacillus moniliformis in a child: human infection and rat carriage diagnosed by PCR. J Clin Pathol. 2005;58:1215-6. 